\title{
SEGMENTATION OF BUILDINGS BASED ON HIGH RESOLUTION PERSISTENT SCATTERER POINT CLOUDS
}

\author{
P. J. Schneider ${ }^{1 *}$ U. Soergel ${ }^{1}$ \\ ${ }^{1}$ Institute for Photogrammetry (ifp), University of Stuttgart, Germany - (philipp.schneider, uwe.soergel)@ifp.uni-stuttgart.de
}

Commission III, WG III/3

KEY WORDS: Persistent Scatterer Interferometry (PSI), Synthetic Aperture Radar, Airborn Laser Scanning, Data Fusion, Clustering, Reverse Geocoding, Time Series Analysis, Building Information Modeling, Data Mining

\begin{abstract}
:
Integrating differential synthetic aperture radar measurements into building information modeling systems requires a mapping of these measurement points onto structural parts of the building. We use a reverse geocoding approach to project building footprints into slant-range geometry, which helps to accurately assign PS points to single building identities. By treating the deformation time series as points in a high dimensional feature space, we can use dimensional reduction and clustering techniques to extract clusters of points that show a similar movement behavior. We visualize these clusters by mapping them onto ground truth, using laser scanning point clouds. Our approach segments buildings into plausible parts.
\end{abstract}

\section{INTRODUCTION}

In the last twenty years the monitoring of deformation processes such as slow landslides, mining induced subsidence and post earthquake tectonic movements has been more and more carried out by interferometric synthetic aperture radar (InSAR) techniques (Hanssen, 2001). When it comes to monitoring urban areas, persistent scatterer interferometry (PSI) (Ferretti et al., 2001) has proven to work reliably and is becoming a standard technique in the portfolio of monitoring. The availability of open space-born SAR data made nation wide deformation mapping services possible. However, the rather low spatial resolution of such data limits their applicability for monitoring at finer scales, for example, motion patterns of individual buildings. High resolution SAR data such as TerraSAR-X are suitable for this task, but come with the downside of a limited acquisition capacity. In the future, upcoming SAR satellite constellations will provide a greater availability of high resolution SAR data, better than $1 \mathrm{~m} \times 1 \mathrm{~m}$ (Farquharson et al., 2018). This enables officials the possibility to efficiently monitor smaller objects of interest, such as critical infrastructure like bridges and dams as well as single buildings.

The results of such monitoring can be either handled isolated or set in relation to other information related to the building. Such building information modeling (BIM) systems use sensors, meters, network infrastructure and 3D modeling to create a digital twin of the structure to inform decisions, such as predictive maintenance (Khajavi et al., 2019). PSI analysis of high resolution SAR data often covers buildings with thousands of derived PSI points in the order of 1 PS per $\mathrm{m}^{2}$ on the façades (Schunert et al., 2012). In the following, we are exploring a method to aggregate and cluster these scatteres into groups of points that show similar movements and therefore are assumed to be located on the same structural part of the building. These parts then could be linked to actual structures of the building via a BIM and allow to augment the information with deformation histories.

\footnotetext{
*Corresponding author
}

Tanaka and Hoshuyama (2017) showed that an evaluation index derived from the correlation of displacement and topography phase shift can be used to extract rigid structures, using minimum spanning trees. A deformation history based clustering was initially used by Zhu et al. (2018) and Costantini et al. (2018), who suggest a clustering algorithm to find temporal deformation patterns in COSMO-SkyMed DInSAR results. By comparison to in-situ ground truth measurements (Schneider et al., 2020) showed that such clusters indeed reflect the actual movement of building parts, but the restricted clustering algorithm could only extract a fraction of potential clusters.

The focus of this work is to refine the allocation of PS points to single buildings and the following clustering algorithm that segments these into independent parts. By combining the results with laser scanning data, we can visualize the extracted building segments and show their plausibility. We also consider this investigation as a preliminary step towards an integration of InSAR based city wide monitoring results into BIM Systems.

In Sec. 2 we describe the SAR and laser scanning database, followed by the methods for label transfer and a detailed description of the clustering approach. Finally we present our results in Sec. 3 along with more examples in the appendix 4.1/4.2. In Sec. 4 we comment our outcome and give an outlook to future directions and applications.

\section{METHODS AND DATABASE}

\subsection{ALS-Data}

We are using airborne laser-scanning (ALS) data to represent the 3-dimensional structure of buildings. The ALS Data has a point density of $40 \mathrm{pts} / \mathrm{m}^{2}$ and a positional accuracy of $\sigma_{X Y Z}=0.02 \mathrm{~m}$. We also derived a digital surface model (DSM) from this point cloud. The DSM served as reference elevation model for the PSI processing and the Reverse Geocoding as described in Sec.2.4, as well as for visualizing the PS-Clusters on the building in Sec. 2.5. 


\subsection{SAR-Data}

The SAR data we are using has been acquired by the German X-Band SAR satellite TerraSAR-X (TSX). The corresponding slant range - azimuth resolution for the "High Resolution Spotlight 300Mhz" acquisition mode is $0.6 \mathrm{~m} \times 1.1 \mathrm{~m}$ (Airbus, 2017). The 132 images were captured during a 4 years time span (September 2016 to October 2020) with 11 days repeat cycle. For the interferogram generation a master image in November 2018 was chosen (Tab. 1). Thus the maximum spatial baseline does not exceed 400m (see Fig. 1).

\subsection{Persistent scatterer interferometry}

The image stack was processed with L3 SARscape's Persistent scatterer interferometry - algorithm (SARMAP, 2020) that is implemented based on Ferretti et al. (2001). For interferogram generation and geocoding, we used a precise surface model, derived from the airborne laser-scanning (ALS) data. As the coherence threshold for the PS-points we choose 0.7 .

Persistent scatterer interferometry (PSI) is an advanced InSAR technique. The main idea of this algorithm is the detection of temporally coherent pixels in a stack of co-registered SAR images. By analyzing the phases of such pixels in each image of the stack, relative to a master image, the line-of-sight (LOS) movement history and a 3D position of this scatterer can be estimated (Ferretti et al., 2001, 2000). Crosetto et al. (2016) give a good overview over the history and the capabilities of PSI algorithms. For more detailed insights we highly recommend reading that article.

PSI works well for dense urban areas, since man-made structures especially metal parts located at house façades and roofs act as a good reflectors (Schunert et al., 2012). The PSI algorithm is able to estimate the scatterer's lateral positions in the order of the pixel size while the height component is typically less accurate (Chang and Hanssen, 2014).

Since PSI is analyzing time series of multiple SAR images, the displacement history of each scatterer is on of the results. For every $P S_{n}$ point we obtain its relative deformation $d_{n}\left(t_{m}\right)$ as a time series with a measurement for each SAR acquisition ( $m \in \mathbb{N} \mid 1 \geq m \leq 132$ ) (see Eq.(1)). The accuracy for each measurement can be better than $2 \mathrm{~mm}$ (Maccabiani et al., 2017).

The deformation time series represent the most advanced PSI product and is the base for the later clustering approach. As Gernhardt et al. (2010) and Crosetto et al. (2015) have shown, PSI time series, derived from high resolution SAR data, are able to reveal the annual movements of buildings. They confirm thermal expansion of buildings up to several millimeters in amplitude over the year. We exploit this fact for our clustering, under the assumption, that each segment of a building exhibits a characteristic movement behavior.

\subsection{Label Transformation}

To assign PS-points to single buildings we used Open Street Maps (OSM) building footprints. Since the PS-algorithm is able to determine a lateral position for each scatterer, a straight forward way to find PS-points in a buildings polygon would be a simple "inpolygon" query for each PS. This approach is shown in Schneider et al. (2020) by considering a buffer around each

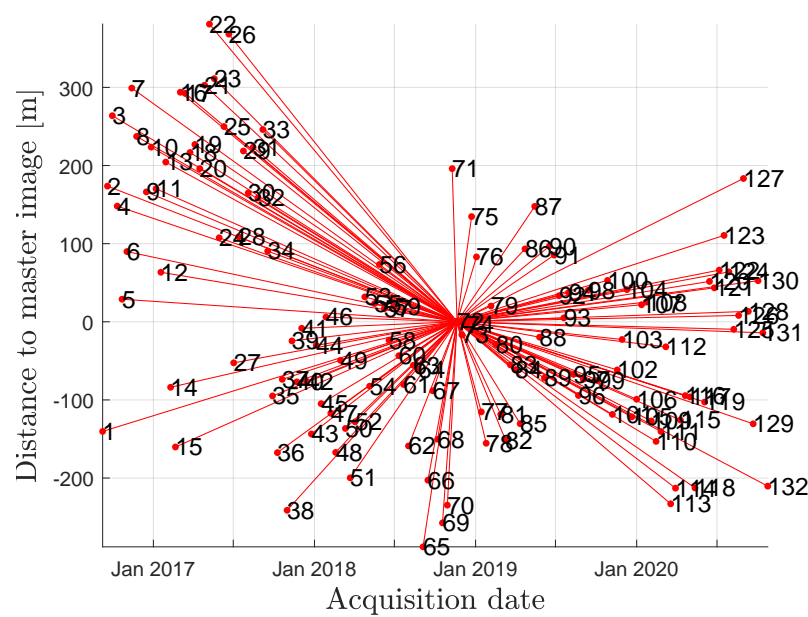

Figure 1. Temporal and spatial baselines, relative to the master image from 20th November 2018. The 132 images were acquired between 8th September 2016 and 24th October 2020, with a repeat interval of 11 days.

\begin{tabular}{|l|l|}
\hline & \\
Platform & TerraSAR-X \\
Wavelength & 31mm (X-Band) \\
Acquisition Mode & HRSL \\
Central Point & $8^{\circ} 47^{\prime} 55^{\prime \prime}$ E9 ${ }^{\circ} 11^{\prime} 33^{\prime \prime}$ \\
Orbit Direction & ASCENDING \\
Resolution: Range $\times$ Azimuth & $0.6 \mathrm{~m} \times 1.1 \mathrm{~m}$ \\
Number of Images & 132 \\
Time interval & $08.09 .2016-24.10 .2020$ \\
Repeat Time & 11 Days \\
PS-Algorithm & L3 SARscape 5.5 \\
Date of Master & 20.11 .2018 \\
Coherence Threshold & 0.7 \\
\hline
\end{tabular}

Table 1. SAR Data acquisition and processing parameters.

building. To overcome the inaccuracy and issues of falsely assigned points we implemented a Reverse Geocoding approach as shown in Fig. 2. The main idea hereby is to utilize an existing pipeline (SARMAP, 2020) to geocode two lookup tables (LuTs). The LuTs contain a unique identifier for each range and azimuth cell. After geocoding with a precise DSM these LuTs are used to transfer labels respectively building footprints into the master images range-azimuth geometry. This allows precise assignment for each PS-point, without the need of the estimated UTM-coordinates. The results as shown in Fig. 3 display a sharp distinguish between neighboring buildings, which would be a challenging task with the inpolygon - buffer method. This approach is also suitable to transform training data into rangeazimuth geometry i.e. for land classification applications.

\subsection{PS- Point clustering}

We treat the deformation histories of each PS point as points in a $M$ dimensional space, with a dimension for each acquisition date. Each point $d_{n} \in \mathbb{R}^{M}$ is defined by the M measurements $d$ as shown Eq. (1).

$$
d_{n}=\left[\begin{array}{lllll}
d_{1}^{n} & d_{2}^{n} & \ldots & d_{m-1}^{n} & d_{m}^{n}
\end{array}\right]
$$

The underlying assumption is that points showing a related movement are close in this deformation space. Schneider et al. 

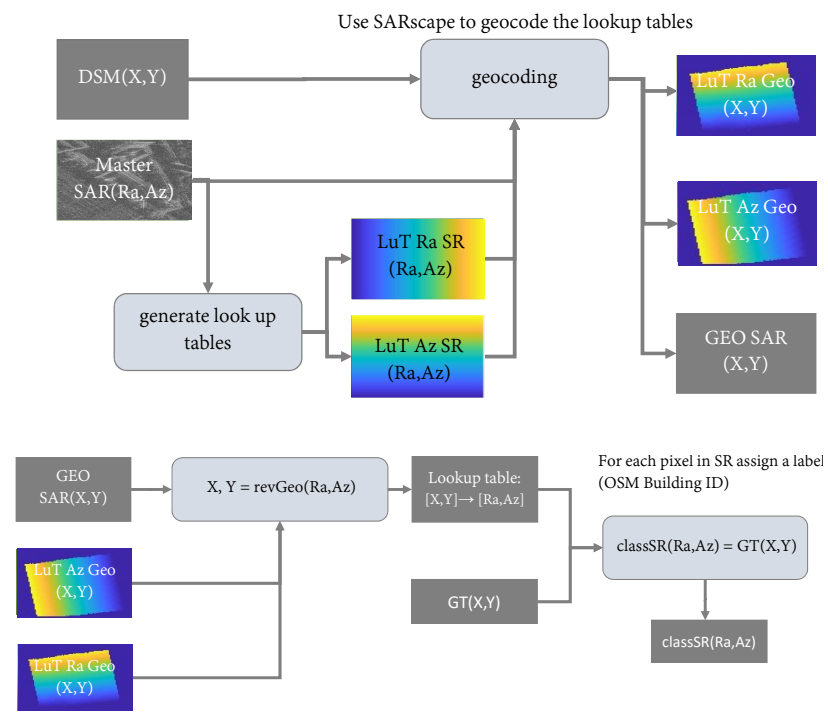

Figure 2. Workflow for Reverse Geocoding: Lookup tables (LuTs) for Range and Azimuth are geocoded, using the master images's SAR properties and a precise digital surface model (DSM). The geocoded LuTs are used to transform the ground truth (GT) from UTM to slant-range geometry, including SAR characteristics like foreshortening and layover.

(2020) have shown for similar data, that "1 minus the sample correlation " (Eq. (2)) is a good heuristic metric for a densitybased spatial clustering of applications with noise (DBSCAN) clustering (Ester et al., 1996) in this space.

$$
\overline{d_{x} d_{y}}=1-\operatorname{corr}\left(d_{a}, d_{b}\right) \quad(\text { see Appx. 4.3) }
$$

Nevertheless these results lead to very sparse clusters with the results being vulnerable to the choice of DBSCAN's $\epsilon$ parameter. Therefore we use a multi step clustering approach:

I t-distributed stochastic neighborhood embedding (t-SNE) (van der Maaten and Hinton, 2008) to reduce the $M$ dimensions drastically to a two-dimensional space. The metric to determine the distance between two PS deformation time series $d_{a}$ and $d_{b}$ for the t-SNE embedding is set to $1-\operatorname{corr}\left(d_{a}, d_{b}\right)$ (Eq. (2)) (Schneider et al., 2020). The resulting two-dimensional representation should preserve the local neighborhoods respectively clusters in the original data.

II Each point in the embedded result is then characterized by a core distance (CD) density estimator (Ankerst et al., 1999). The core distance of a point is the smallest threshold such that the point is still considered a core object by the DBSCAN algorithm.

III We then exclude all points where the $\mathrm{CD}>$ median(CD).

IV The remaining embedded points are now clustered with DBSCAN $(\epsilon=\operatorname{median}(\mathrm{CD}) \cdot 1.5$; minPts $=5)$. For each resulting DBSCAN cluster we calculate the center point $\bar{d}_{k}=$ mean $\left(d_{\text {all }}\right)$ in deformation space.

$\mathrm{V}$ Now we discard outliers by comparing each cluster point to the center using again the $1-\operatorname{corr}\left(\overline{d_{k}}, d_{i}\right)$ distance metric and a correlation threshold of $C_{t}=0.3$.
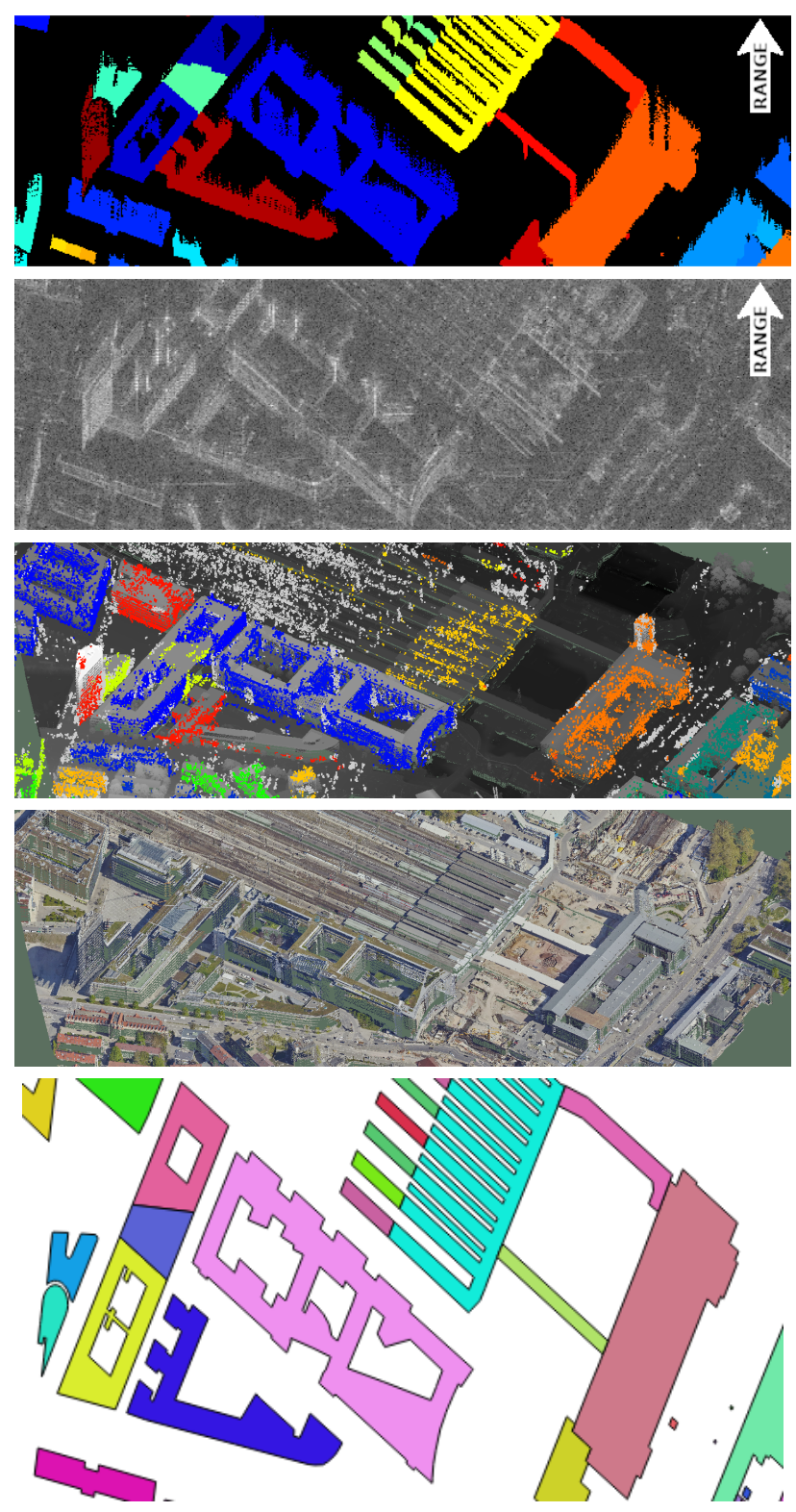

Figure 3. Result of Reverse Geocoding: from top to bottom: Building instances in slant-range geometry (SL); Amplitude SAR image in SL; 3d view of DSM with PS-points (one color per building); 3d view of RGB ALS point cloud; Building footprints from Open Street Map.

VI Finally we merge similar clusters by comparing there center points $\bar{d}_{j}$ and $\bar{d}_{k}$ and merge the cluster $k$ with cluster $j$ if $1-\operatorname{corr}\left(\bar{d}_{k}, \bar{d}_{j}\right)<C_{t}$.

The result of this consecutive clustering process are grouped PS-points that show homogeneous movement. In contrast to Schneider et al. (2020), the resulting clusters are less sparse and we can assign more than $80 \%$ of the PS-points to a cluster. The workflow, including exemplary interim results for each step, is shown in Fig. 4. To visualize the clusters on the building we are assigning each ALS-point to the closest PS-cluster via nearest neighbor, with a maximum distance of $10 \mathrm{~m}$. The center points of each cluster in deformation space can be interpreted as the clusters mean movement over time. 


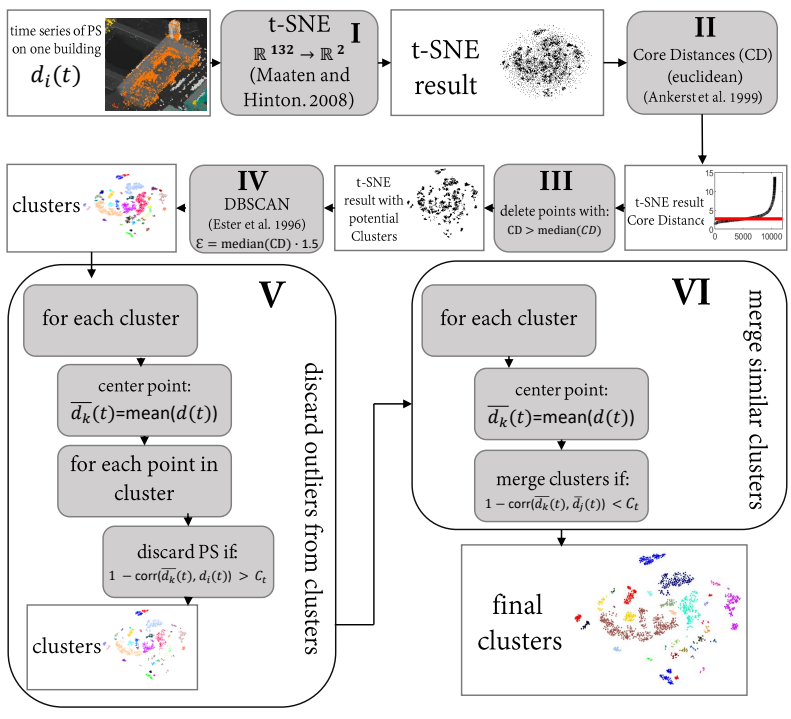

Figure 4. Schematic workflow of the clustering process. The $M$ dimensional PS-points on a single building are embedded into a 2D space via t-SNE. For all points in this embedding the core distance $C D$ is estimated. Points that have a $C D$ greater than the overall median are then excluded. DBSCAN is then performed on the remaining points. For each point in each cluster the distance to the clusters center of gravity in the original $M$ dimensional deformation space is then calculated. Based ob this metric points are excluded from a cluster if their distance is greater than a correlation threshold $\left(C_{t}=0.3\right)$. In a final step clusters are merged if their center of gravity is closer than the $C_{t}$.

\section{RESULTS}

For this study we evaluated several buildings with our method. In Fig. 5 we show a colored ALS point cloud. Each color represents a cluster. One can see that the PS-clusters divide the building into sections. This is especially remarkable since no spatial information about the PS-points location is considered in the clustering process. For instance, the tower in Fig. 5 is clearly divided into two clusters. The corresponding t-SNE plot is given in Fig.6. Our clustering approach was able to identify most of the visible clusters while distinguishing the independent, non-cluster points. The corresponding time-series for each cluster are presented in Fig. 7, along with the daily average temperature. The central point of each cluster is colored emphasized by color. In appendix 4.1A and 4.2B we included two more examples of segmented buildings. Both show a reasonable segmentation into sub parts.

\section{CONCLUSION}

We apply a reverse geocoding approach to map PS points onto OSM building footprints to accurately identify all scatteres on a single building. We used the deformation histories derived from high resolution SAR PSI analysis to extract clusters, which are groups of PS points that show a similar displacement behavior over time. We visualized the results by ALS point clouds of the buildings. The clusters on the structures show plausible segments of a the buildings, even though no spatial information

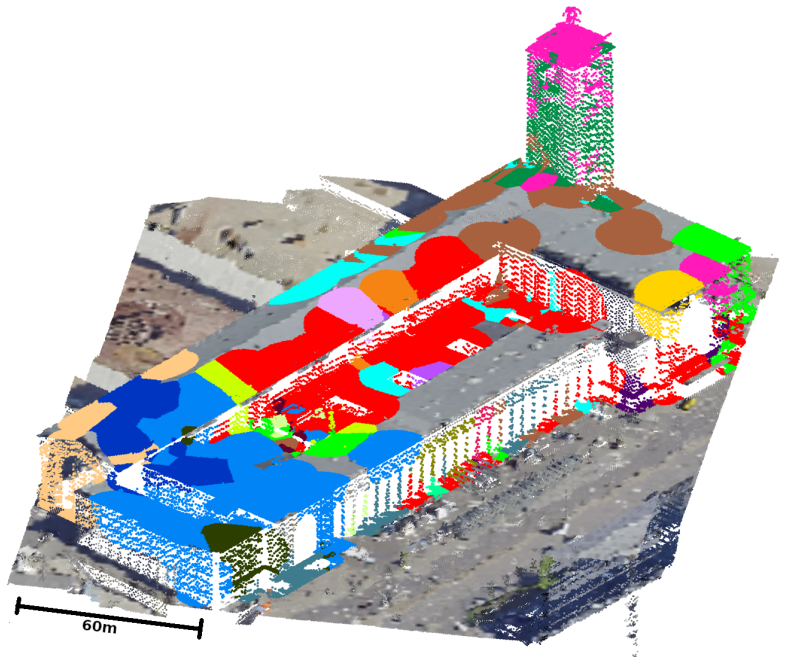

Figure 5. ALS point cloud colored with the resulting clusters on Building 1. Each color corresponds to a cluster (Fig. 6) and a time series in Figure 7.

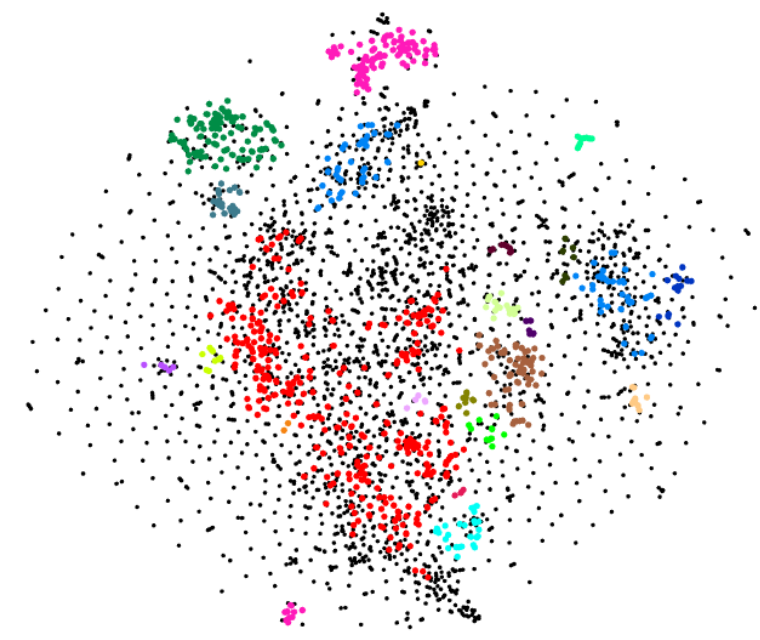

Figure 6. t-SNE embedding of the deformation space with clusters. The colors correspond to the ALS point cloud (Fig. 5) and the time series in Figure 7.

has been considered in the clustering process. The here shown data set consists of quite a high number of images (132) since the acquisition of such data can be very expensive it is worth to mention that previous experiments on shorter time series $(30+$ images) achieve similar results to some extend.

Our future and ongoing work is focusing to map such clusters onto building components provided by BIMs. Another interesting application of these clusters can be a risk assignment and stress prediction by comparing the movements of neighboring clusters.

\section{ACKNOWLEDGEMENTS}

The TerraSAR-X data were provided by the German Aerospace Center (DLR) through the proposal LAN0634. We would like to thank the State Office for Spatial Information and Land Development Baden-Württemberg (LGL) for providing citywide ALS data as well as high resolution orthophotos to colorize 


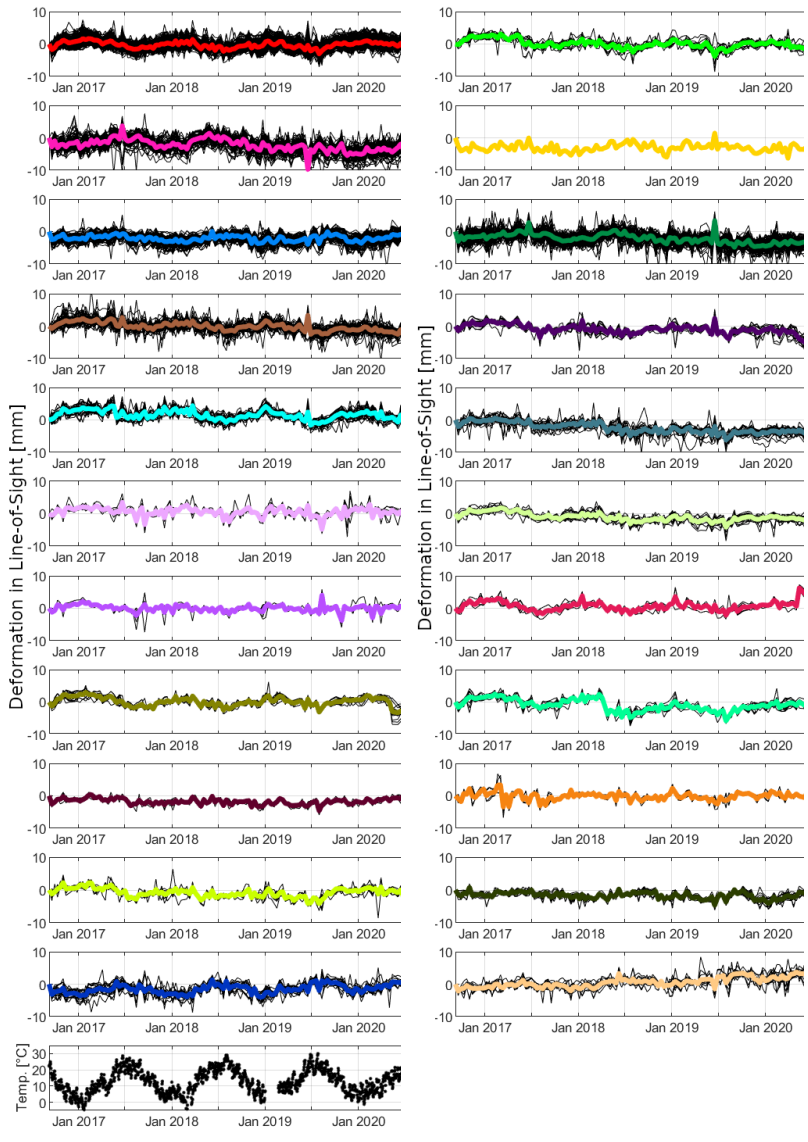

Figure 7. Time series for each cluster. The colors correspond to the ALS point cloud (Fig. 5) and the t-SNE plot (Fig. 6). The daily temperature is shown in the lower left plot

these points. The temperature data was provided by Amt für Umweltschutz, Stadt Stuttgart.

\section{REFERENCES}

Airbus, 2017. TerraSAR-X Image Product Guide Basic and Enhanced Radar Satellite Imagery. ( February 2020). https://www.intelligence-airbusds. com/files/pmedia/public/r459_9_20171004_ tsxx-airbusds-ma-0009_tsx-productguide_i2.01.pdf.

Ankerst, M., Breunig, M. M., Kriegel, H.-P. and Sander, J., 1999. OPTICS. ACM SIGMOD Record 28(2), pp. 49-60.

Chang, L. and Hanssen, R., 2014. Detection of cavity migration and sinkhole risk using radar interferometric time series. Remote sensing of environment 147, pp. 56-64.

Costantini, M., Zhu, M., Huang, S., Bai, S., Cui, J., Minati, F., Vecchioli, F., Jin, D. and Hu, Q., 2018. Automatic detection of building and infrastructure instabilities by spatial and temporal analysis of insar measurements. IGARSS 2018 2018 IEEE International Geoscience and Remote Sensing Symposium pp. 2224-2227.

Crosetto, M., Monserrat, O., Cuevas-González, M., Devanthéry, N. and Crippa, B., 2016. Persistent scatterer interferometry: A review. ISPRS Journal of Photogrammetry and Remote Sensing 115, pp. 78-89.
Crosetto, M., Monserrat, O., Cuevas-González, M., Devanthéry, N., Luzi, G. and Crippa, B., 2015. Measuring thermal expansion using X-band Persistent Scatterer Interferometry. ISPRS Journal of Photogrammetry and Remote Sensing (accepted for publication) 100, pp. 84-91.

Ester, M., Kriegel, H.-P., Sander, J. and Xu, X., 1996. A density-based algorithm for discovering clusters a densitybased algorithm for discovering clusters in large spatial databases with noise. In: Proceedings of the Second International Conference on Knowledge Discovery and Data Mining, KDD'96, AAAI Press, pp. 226-231.

Farquharson, G., Woods, W., Stringham, C., Sankarambadi, N. and Riggi, L., 2018. The capella synthetic aperture radar constellation. In: IGARSS 2018 - 2018 IEEE International Geoscience and Remote Sensing Symposium, IEEE.

Ferretti, A., Prati, C. and Rocca, F., 2000. Nonlinear subsidence rate estimation using permanent scatterers in differential sar interferometry. Geoscience and Remote Sensing, IEEE Transactions on 38 , pp. $2202-2212$.

Ferretti, A., Prati, C. and Rocca, F., 2001. Permanent scatterers in SAR interferometry. IEEE Transactions on Geoscience and Remote Sensing 39(1), pp. 8-20.

Gernhardt, S., Adam, N., Eineder, M. and Bamler, R., 2010. Potential of very high resolution sar for persistent scatterer interferometry in urban areas. Annals of GIS 16, pp. 103-111.

Hanssen, R., 2001. Radar Interferometry Data Interpretation and Error Analysis. Springer.

Khajavi, S. H., Motlagh, N. H., Jaribion, A., Werner, L. C. and Holmstrom, J., 2019. Digital twin: Vision, benefits, boundaries, and creation for buildings. IEEE Access 7, pp. 147406-147419.

Maccabiani, J., Nicodemo, G., Peduto, D. and Ferlisi, S., 2017. Investigating building settlements via very high resolution sar sensors. In: Proceedings of the Fifth International Symposium on Life-Cycle Civil Engineering, IALCCE 2016, p. 8.

SARMAP, 2020. Sarscape: Technical description. SARMAP: Purasca.

Schneider, P. J., Khamis, R. and Soergel, U., 2020. EXTRACTING AND EVALUATING CLUSTERS IN DINSAR DEFORMATION DATA ON SINGLE BUILDINGS. ISPRS Annals of Photogrammetry, Remote Sensing and Spatial Information Sciences V-3-2020, pp. 157-163.

Schunert, A., Schack, L. and Soergel, U., 2012. Matching Persistent Scatterers to Buildings. ISPRS - International Archives of the Photogrammetry, Remote Sensing and Spatial Information Sciences XXXIX-B7, pp. 79-84.

Tanaka, T. and Hoshuyama, O., 2017. Persistent scatterer clustering for structure displacement analysis based on phase correlation network. In: 2017 IEEE International Geoscience and Remote Sensing Symposium (IGARSS), IEEE.

van der Maaten, L. and Hinton, G., 2008. Viualizing data using t-sne. Journal of Machine Learning Research 9, pp. 2579-2605.

Zhu, M., Wan, X., Fei, B., Qiao, Z., Ge, C., Minati, F., Vecchioli, F., Li, J. and Costantini, M., 2018. Detection of building and infrastructure instabilities by automatic spatiotemporal analysis of satellite sar interferometry measurements. Remote Sensing 10(11), pp. 1816. 


\section{APPENDIX}

4.1 A
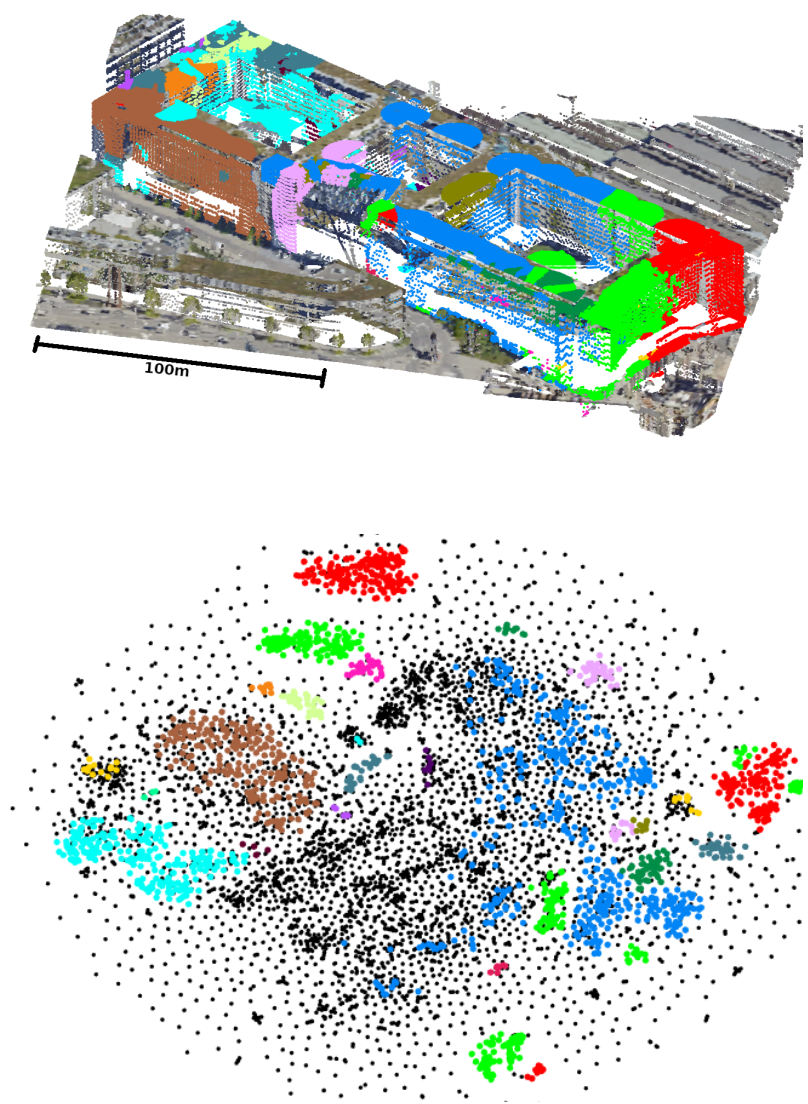

Figure 8. Top: ALS point cloud with colored clusters on Building A. Bottom: Corresponding t-SNE embedding. The related time series are given in Figure 9.

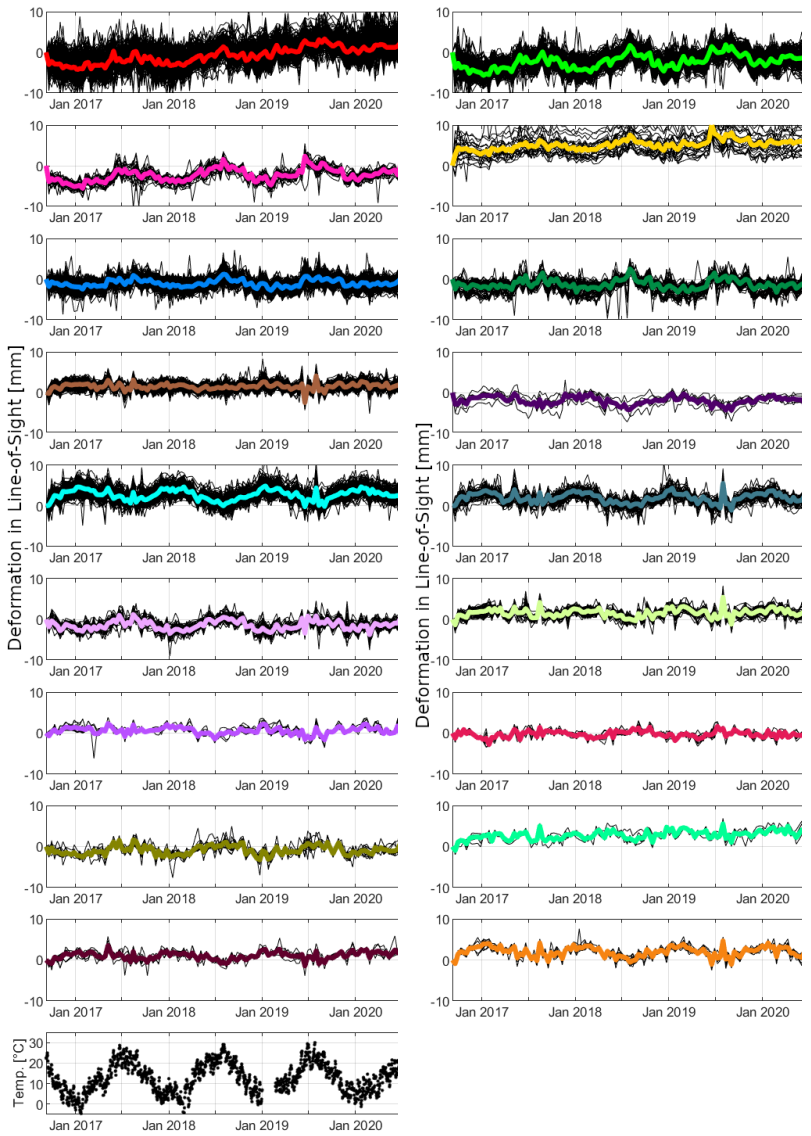

Figure 9. Time series of clusters on building A. The center point of each cluster is plotted in color. The colors correspond to the clusters in Figure 8. The bottom plot shows the mean daily temperature. 

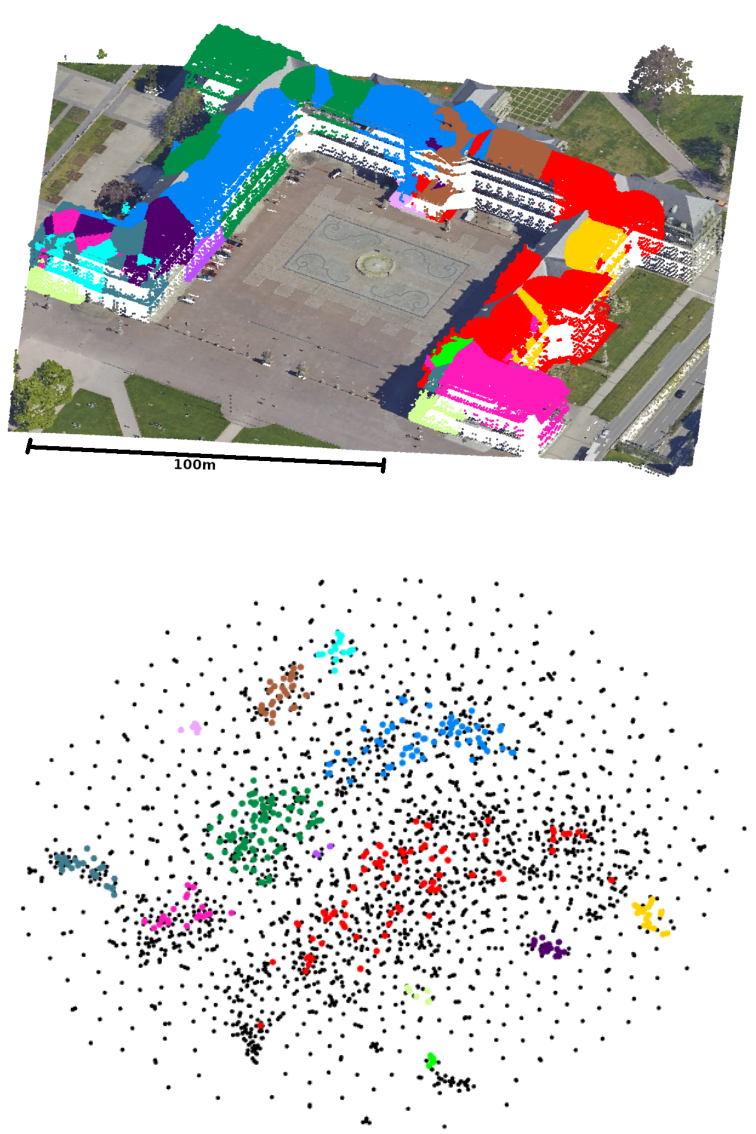

Figure 10. Top: ALS point cloud with colored clusters on Building B. Bottom: Corresponding t-SNE embedding. The related time series are given in Figure 11.
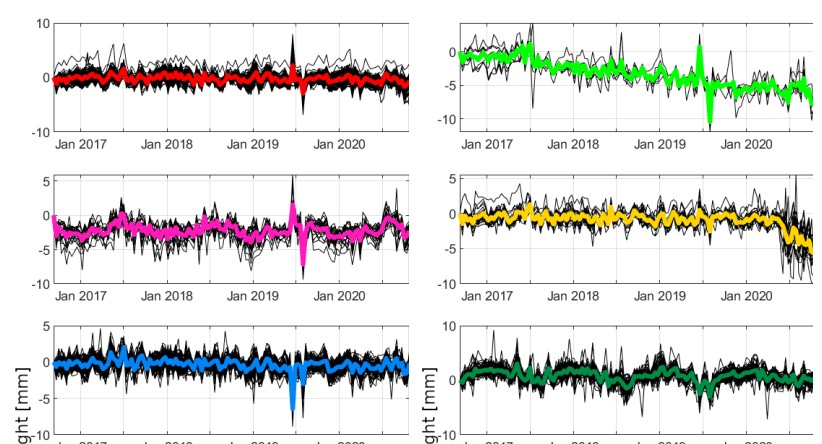

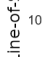
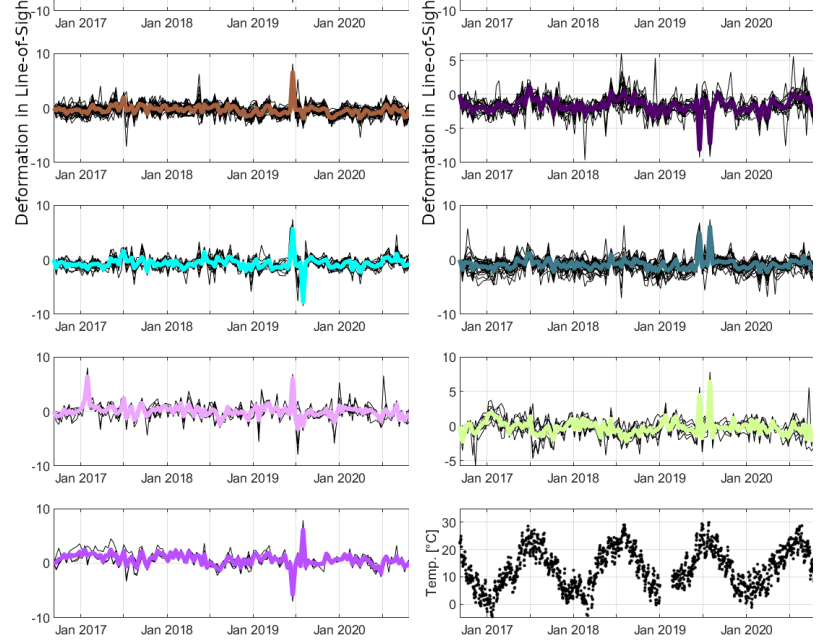

Figure 11. Time series of clusters on building B. The center point of each cluster is plotted in color. The colors correspond to the clusters in Figure 10. The bottom plot shows the mean daily temperature. We are not sure about the origin of the "spikes" around June 2019. They could be processing artifacts due to atmospheric influence.

\subsection{Distance metric}

"1- minus the sample correlation" distance metric for two PSpoints $d_{x}$ and $d_{y}$ in $M$ dimensional deformation space:

$$
\begin{gathered}
\overline{d_{x} d_{y}}=1-\operatorname{corr}\left(d_{a}, d_{b}\right) \\
=1-\frac{\left(d_{x}-\overline{d_{x}}\right)\left(d_{y}-\overline{d_{y}}\right)^{T}}{\sqrt{\left(d_{x}-\overline{d_{x}}\right)\left(d_{x}-\overline{d_{x}}\right)^{T}} \cdot \sqrt{\left(d_{y}-\overline{d_{y}}\right)\left(d_{y}-\overline{d_{y}}\right)^{T}}} \\
\text { where } \quad \overline{d_{x}}=\frac{1}{M} \sum_{m}^{M} d_{m}^{x} \\
\overline{d_{y}}=\frac{1}{M} \sum_{m}^{M} d_{m}^{y} \\
\text { and } d_{x / y} \text { as in Eq. (1). }
\end{gathered}
$$

Revised January 2021 